\title{
RECENT STUDIES OF DISPERSION MATCHED STEERING FOR THE ILC BUNCH COMPRESSOR AND MAIN LINAC *
}

\author{
Paul L. G. Lebrun, Leo Michelotti and Jean-François Ostiguy \\ Fermilab, IL 60510, USA $^{\dagger}$
}

\begin{abstract}
The Dispersion Matched Steering (DMS) method is studied in detail in the context of a curved main linac. In the absence of cavity tilts (rotations in the YZ plane), DMS provides a unique and stable solution with negligible emittance growth. If cavity tilts are about $300 \mu \mathrm{rad}$, the algorithm is not very robust. The emittance growth through the entire linac for positrons is about $5 \mathrm{~nm}$, if the system is strictly static and statistical averaging can be used to improve beam position measurements. This growth is mostly eliminated if the dispersion and its derivative at injection can be adjusted. If anticipated ground motion, beam and klystron jitter, beam position measurement resolution are introduced (i.e. dynamical case), the emittance preservation goal is currently not achieved by DMS alone. Mitigation strategies are outlined.
\end{abstract}

\section{INTRODUCTION}

Transverse emittance preservation from the damping ring to the interaction point is a critical item on the list of challenges for the International Linear Collider (ILC)[1]. The Low Emittance Transport (LET) problem has been the main focus of the ILC Accelerator Physics (APY) group. Basic steering methods aimed at preserving the r.m.s transverse emittance through a misaligned linac are by now well-developed [2, 3, 4]. Nevertheless, numerous details still need to be studied in order to meet the challenging goal of limiting the total vertical emittance dilution to a few nanometers in a linac that follows the earth's curvature [5].

This paper discusses the performance of the Dispersion Matched Steering algorithm[5], focusing on (i) convergence and robustness in the presence of tilted and misaligned cavities (ii) the impact of dynamical perturbations, such as beam jitter at injection, klystron jitter, vibrations, and ground motion. Although it reports on detailed simulations of the ILC main linac (ML), our results are also relevant to the bunch compressors, where emittance preservation is more difficult because of lower beam momentum. The lattice used here corresponds to an early version of the ILC Reference Design Report[7]. Although the ML lattice has been refined since, basic features such as betatron phase advance, curvature and accelerating gradient have remained unchanged. Alignment tolerances are assumed to be those agreed upon in the ILC/APY group, except that

\footnotetext{
${ }^{*}$ Work by Fermi Research Alliance, LLC under Contract No. DEAC02-07CH11359 with the United States Department of Energy.

† lebrun@fnal.gov
}

a survey monument placement error of $200 \mu \mathrm{m}$ every $\mathrm{km}$ has been introduced ${ }^{1}$. In this study, only emittance preservation in the vertical plane is considered. This emittance at injection is only $20 \mathrm{~nm}$. Coupling between the horizontal and vertical planes (e.g. from quadrupole rotations) is ignored.

In the absence of accelerating structure misalignments, wakefields, ground motion etc., an estimate for the emittance growth after optimum steering can be obtained using analytical methods[2]. To account for such effects, one must resort to numerical techniques. We use the CHEF[8] framework, which has been bench-marked sucessfully against other codes used within the LET collaboration, thereby providing independent confirmation of previous results.

\section{DISPERSION MATCHED STEERING}

"Ballistic Alignment"[6] is a straightforward beambased alignment technique proposed for linear colliders where beam position monitor (BPM) offsets with respect to the beam line and quadrupole centers are determined. Partial focusing, i.e., "quad-shunting" is a complementary way of determining these offsets. However, in both cases, one must assume that the magnetic center does not depend on the excitation of these magnets. This is unfortunately not always true.

An alternative method is based on the measurement and minimization of the residual dispersion $\left(D_{y}\right)$, which can be estimated based on beam position differences resulting from momentum changes. For a straight linac, this is referred to as the Dispersion Free Steering method, as the optimum $D_{y}$ vanishes. Steering towards such a null quantity is advantageous since no absolute scale is involved. The case of a curved linac is more complicated because it requires knowledge of both the optimum $D_{y}$ and the "scale" of the BPM. The latter is defined as the ratio between a change in position and the corresponding change in the readout[5].

In this study, the salient features of DMS are:

- Steering is done section by section, to keep corrections local. The length of a DMS section is 20 dipoles $(\approx 3.3$ betatron wavelengths). The overlap between sections is 10 dipoles. A complete steering iteration is done when the last, most downstream DMS section is tuned.

\footnotetext{
${ }^{1}$ This is a minor refinement which does not invalidate any previously reached conclusions.
} 
- The accelerating gradients in a given DMS section for the on-momentum and off-momentum beams are equal. This implies that an upstream device in the main linac, or the bunch compressor, is used to change the beam energy.

- The $\Delta p / p$ used to measure $D_{y}$ should be as small as possible to avoid non-linearities. BPM scale errors and klystron output power stability set a lower bound on its value. A preliminary value of $\Delta p / p$ of 2 to $3 \%$ has been chosen.

- The momentum is varied by changing the accelerating voltage. Operationally, it is simpler to change the phase but this affects the momentum spread. The issue deserves further study.

- The relative magnitudes of the BPM to quadrupole center offsets $(175 \mu \mathrm{m})$ and the dispersion measurement error $(4 \mu \mathrm{m})$ are used to establish the weighting in the DMS algorithm's objective function ( $w_{0}$ and $w_{1}$ used in equation (2) of ref. [5]).

- To avoid numerical instabilities, a suitable subspace of the linear response matrix associated with small singular values is suppressed.

- No uncertainty is assigned to the designed, or "target lattice" $D_{y}$ function.

- Multiple pulses are required to average over transients (dynamical perturbations). To improve convergence, only half of the calculated change to each dipole correctors setting is applied at each iteration. The process stops when either the difference between two successive changes is below threshold, or if a specified maximum number of iterations is reached.

- In the dynamic case, a pulse to pulse, r.m.s. BPM resolution of $0.5 \mu \mathrm{m}$ is assumed (Gaussian distributed). ${ }^{2}$

- BPM scale errors are supported in the software but set to zero for the results shown below.

\section{CAVITY DISPLACEMENTS AND TILTS}

If the cavities are perfectly aligned, one solution stands out as straightforward and robust: each dipole corrector located very close to its associated quadrupole exactly compensates for the quadrupole displacement. This solution is "hyper-local," the beam is on axis at the cavities, and in the absence of higher multipole moments in these magnets the emittance is preserved. ${ }^{3}$ This is not true if residual $D_{y}$ is generated by misaligned cavities: one must then either precorrect or post-correct these kicks. Experience shows that linear optics remains adequate to determine the corrections.

Not all aspects of the LET calculation are linear. In particular, the transport matrices through cavities can be expanded in terms of $\Delta \gamma / \gamma$, where $\gamma$ is the usual Lorentz boost factor, and $\Delta \gamma$ is the boost in a single cavity. Here, keeping only the first order was found insufficient at 5 $\mathrm{GeV} / \mathrm{c}$, with a nominal ILC gradient of $31.5 \mathrm{MeV} / \mathrm{m}$. (A

\footnotetext{
${ }^{2}$ Under static assumptions, fluctuations in BPM readings can be averaged out by simply requiring enough pulses. This is not simulated.

${ }^{3}$ This has been studied and found to be not a concern.
}

$1.5 \mathrm{~nm}$ discrepancy in final emittance between Merlin[5] and CHEF was traced to that issue.) This prompted the CHEF team to document and further improve the propagators through such cavities.

In addition, nonlinearities in wakefield kicks may be significant in some situations. Currently, only the dipole mode is used in estimating a transverse kick from the wake potential. Wakefields arising from the presence of couplers are ignored. Systematic errors are therefore hard to quantify.

\section{PERFORMANCE OF DMS ON THE POSITRON MAIN LINAC}

\section{Static case}

A machine at rest, with perfectly stable beam is considered. In the absence of misalignments, emittance growth through the entire ML is less than a fraction of a nm. With realistic misalignments and no steering, a beam with $20 \mathrm{~nm}$ emittance at injection typically emerges from the end of the curved linac with an emittance of $150 \mu \mathrm{m}$. The unwanted trajectory excursions reach several $\mathrm{mm}$ (up to $1 \mathrm{~cm}$ ). To protect the machine, steering will be commissioned using bunches with low bunch charge or without bunch compression. In this case, short range wakefields ${ }^{4}$ are neglected. Reasonable performance is then obtained via DMS alone: the calculated average emittance growth is about $2.5 \mathrm{~nm}$. When the nominal intensity bunches $\left(2 \times 10^{10}\right.$ positrons, $300 \mu \mathrm{m}$ long) are propagated through the corrected linac a typical - and unacceptable - emittance growth of $\approx 6$ to $10 \mathrm{~nm}$ (shown in figure 1) is observed. Since this is a static simulation, little improvement is achieved with multiple complete DMS iterations. Rerunning DMS at nominal charge is required to reduce further the emittance dilution (not shown in the figure). One can also readjust $D_{y}$ and $D_{y}^{\prime}$ at injection; the performance reached with this non-local correction is adequate.

\section{Dynamic Case}

To illustrate the difficulty of controlling the DMS procedure under realistic conditions, i.e. with dynamic perturbations, Figure 2 shows the LET performance under the following assumptions:

- The BPM resolution is $0.5 \mu \mathrm{m}$.

- For each iteration on a DMS section, five successive pulses are used for every position measurement. This is done for both on and off momentum conditions. New dipole corrector settings can be applied in between DMS iterations. Following the tuning of each DMS sections, the procedure is paused for 30 seconds.

- Ground motion corresponds to a moderately quiet site, such as the NUMI tunnel at Fermilab.

- Beam jitter at injection of about $1 \mu \mathrm{m}$ in vertical position and $0.1 \mu$ radians in angle.

\footnotetext{
${ }^{4}$ Multi-bunch, e.g. long range wakefields are not considered at all in this study.
} 


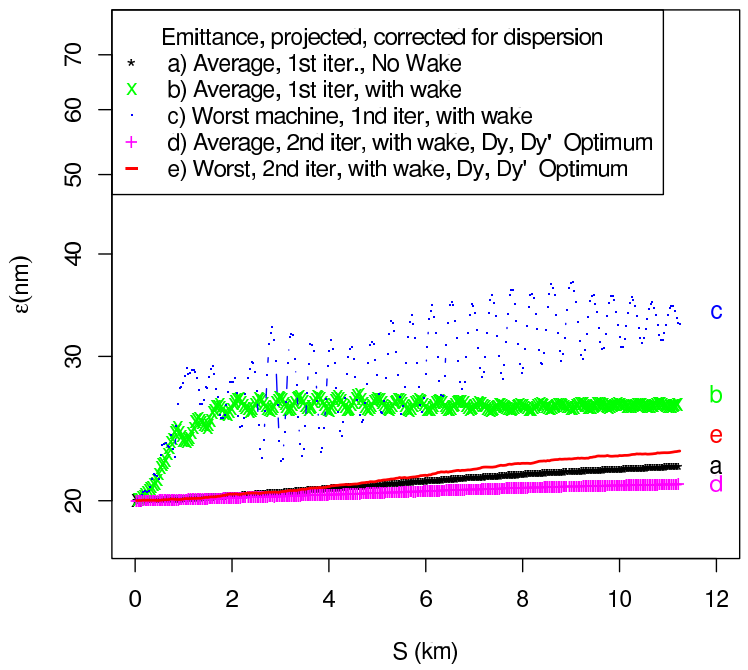

Figure 1: Static LET performance: the average over 99 misaligned machine instances of the vertical, normalized, emittance corrected for dispersion. Also shown is the performance for the worst machine, among the set for which the averages has been estimated.

- Pulse-to-pulse klystron output power fluctuations are neglected.

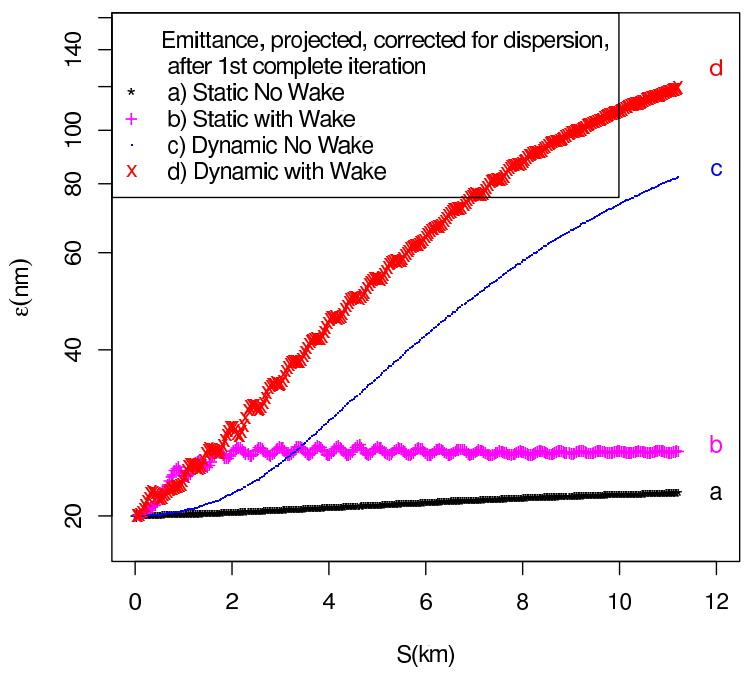

Figure 2: The dynamic performance is compared to the static case.

\section{CONCLUSION}

The performance of the Dispersion Matched Steering algorithm applied to emittance preservation in the ML has been studied. In the static case, emittance dilution satisfies the ILC requirements. This conclusion agrees with previous independent studies. In the dynamic case, DMS alone is likely to be inadequate. Further study of its performance under realistic dynamic conditions is needed. Should the DMS algorithm prove inadequate, the following mitigation strategies are suggested:

- Implement a 5Hz feedback trajectory stabilization[9] for upstream sections, while DMS tuning in downstream sections.

- Estimate the cavity misalignments by instrumenting the High Order Mode couplers[10].

- Reduce the cavity alignment tolerances. If not possible, investigate the possibility of having some cavities on movers.

- To better characterize cavity kicks in the critical first DMS section, consider using a dedicated low energy $(\approx 0.5 \mathrm{GeV})$ injector, with associated transfer line into the $5 \mathrm{GeV}$ bunch compressor. Note that low emittance and short bunches are not needed here, as one simply would investigate and mitigate predominantly dispersive effects.

- Mitigate quadrupole vibrations by improving support stabilization.

\section{REFERENCES}

[1] G. Loew et al ILC-TRC 2003 Report http://www.slac.stanford.edu/xorg/ilctrc/2002/2002/report/03rep.htm

[2] Chris Adolphsen and Tor Raubenheimer, "Method to Evaluate Steering and Alignment Algorithms for Controlling Emittance Growth", PAC'93, Washington DC, May 17-20, 1993.

[3] G.V. Stupakov "Quadrupole Misalignments and Steering in Long linacs" XX International LINAC Conf., Monterey, California.

[4] Jeffrey C. Smith, Dissertation thesis

[5] P. Eliasson et al "Study of an ILC Main Linac Curvature that follows the Earth Curvature", EPAC'06, Edinburgh, Scotland, June 26-30 2006.

[6] T.O. Raubenheimer and D. Schulte "The Ballistic Alignment Method", PAC'99, New York City, U.S.A. March 29 April 2, 1999.

[7] The ILC Reference Design Report, at http://media.linearcollider.org/rdr_draft_v1.pdf

[8] J.-F. Ostiguy and L. Michelotti "CHEF: A framework for Accelerator Optics and Simulation", ICAP-2006, Chamonix, France

[9] V. Ivanov, "Dynamic Steering Algorithms with Taking Into Account the Ground Motion", this conference.

[10] Stephen Molloy em et al "Measurement of the Beam's Trajectory Using the Higher Order Modes it Generates in a Superconducting Accelerating Cavity", this conference. 